\title{
HALLAZGO DE LAURELIOPSIS PHILIPPIANA (LOOSER) SCHODDE (ATHEROSPERMATACEAE) EN EL AREA COSTERA DE LA REGION DEL MAULE, CHILE CENTRAL
}

\author{
OCCURRENCE OF LAURELIOPSIS PHILIPPIANA (LOOSER) SCHODDE \\ (ATHEROSPERMATACEAE) IN THE COASTAL AREA OF THE MAULE \\ REGION, CENTRAL CHILE
}

We report the finding of Laureliopsis philippiana (Looser) Schodde (Atherospermataceae) in the coastal area of the Maule Region ( $35^{\circ} 29^{\prime} \mathrm{S}$ and $72^{\circ} 22^{\prime} \mathrm{W}$ ) in central Chile. The new locality (San Pedro - Las Cañas) extends by some 240 $\mathrm{km}$ the northern limit of the species, which is associated mainly with the temperate rainforests further south.

\author{
César A. Sepúlveda ${ }^{1} \&$ Alejandro Troncoso A². \\ ${ }^{1}$ Comité Nacional Pro-Defensa de la Fauna y Flora (CODEFF). 2 Sur 606, Talca, Chile \\ E-mail: probos.maule@terra.cl \\ ${ }^{2}$ Instituto de Biología Vegetal y Biotecnología. Universidad de Talca. Casilla 747, Talca, Chile
}

Laureliopsis philippiana (Looser) Schodde, "tepa", es uno de los árboles principales del bosque lluvioso valdiviano que se desarrolla entre $38^{\circ} 30^{\prime}$ y $43^{\circ} 20^{\prime}$ $\mathrm{S}$ en el sur de Chile (Kalin-Arroyo et al. 1996). La presente nota informa el hallazgo de una población de esta especie en la zona de clima mediterráneo de Chile central, precisamente en el sector de San Pedro-Las Cañas, vertiente occidental de la Cordillera de la Costa de la Región del Maule (35²9’S y $\left.72^{\circ} 22^{\prime} \mathrm{O}\right)$, a $410 \mathrm{~m}$ snm. Este sitio se ubica a aproximadamente $240 \mathrm{~km}$ al norte de la Cordillera de Nahuelbuta, antiguo límite descrito para esta especie (Rodríguez \& Quezada, 2001). Este descubrimiento viene a sumarse a los de otras especies informados por Troncoso \& San Martín (1988) y por Sepúlveda \& Stoll (2003), cuyas distribuciones geográficas reportadas no incluían la Región del Maule, con lo cual se va configurando una situación de gran interés desde la perspectiva biogeográfica y de la singularidad del bosque maulino.

La población de L. philippiana se encuentra en una unidad fragmentaria (aproximadamente 690 ha) de Nothofagus obliqua (Mirb.) Oerst. y Nothofagus glauca (Phil.) Krasser. Sus individuos se hallan dispersos a orillas de un curso de agua en una quebrada con orientación $\mathrm{O}-\mathrm{E}$, de 1,5 km de extensión y situado $4 \mathrm{~km}$ al norte del río Pinotalca. Los individuos de L. philippiana allí presentes exhiben alturas que varían desde 5 a $15 \mathrm{~m}$ y diámetros de hasta $25 \mathrm{~cm}$. Se observaron frutos inmaduros en las copas de los árboles y abundante regeneración de origen sexuada, lo que indicaría que la población no presenta problemas de reproducción y establecimiento. Las especies arbóreas acompañantes más frecuentes son Nothofagus dombeyi (Mirb.) Oerst., Pitavia punctata Molina y Aextoxicon punctatum Ruiz et Pav.

Actualmente el sector forma parte de un área de conservación denominada "Los Hualos de Loanco", propiedad de la empresa Forestal Copihue. La vegetación circundante está conformada principalmente por plantaciones forestales de Pinus radiata D. Don.

El material foliar estudiado fue diafanizado mediante la técnica propuesta por Barrera (1981) e identificado siguiendo las claves de Barrera \& Meza (1992) y de Rodríguez \& Quezada (2001), constatándose la presencia de pelos malpighiáceos y escamas peltadas en la cara abaxial. Posteriormente, se logró colectar frutos, cuyos pedicelos florales median 1,5-3,5 $\mathrm{mm}$ de largo (Fig. 1). 


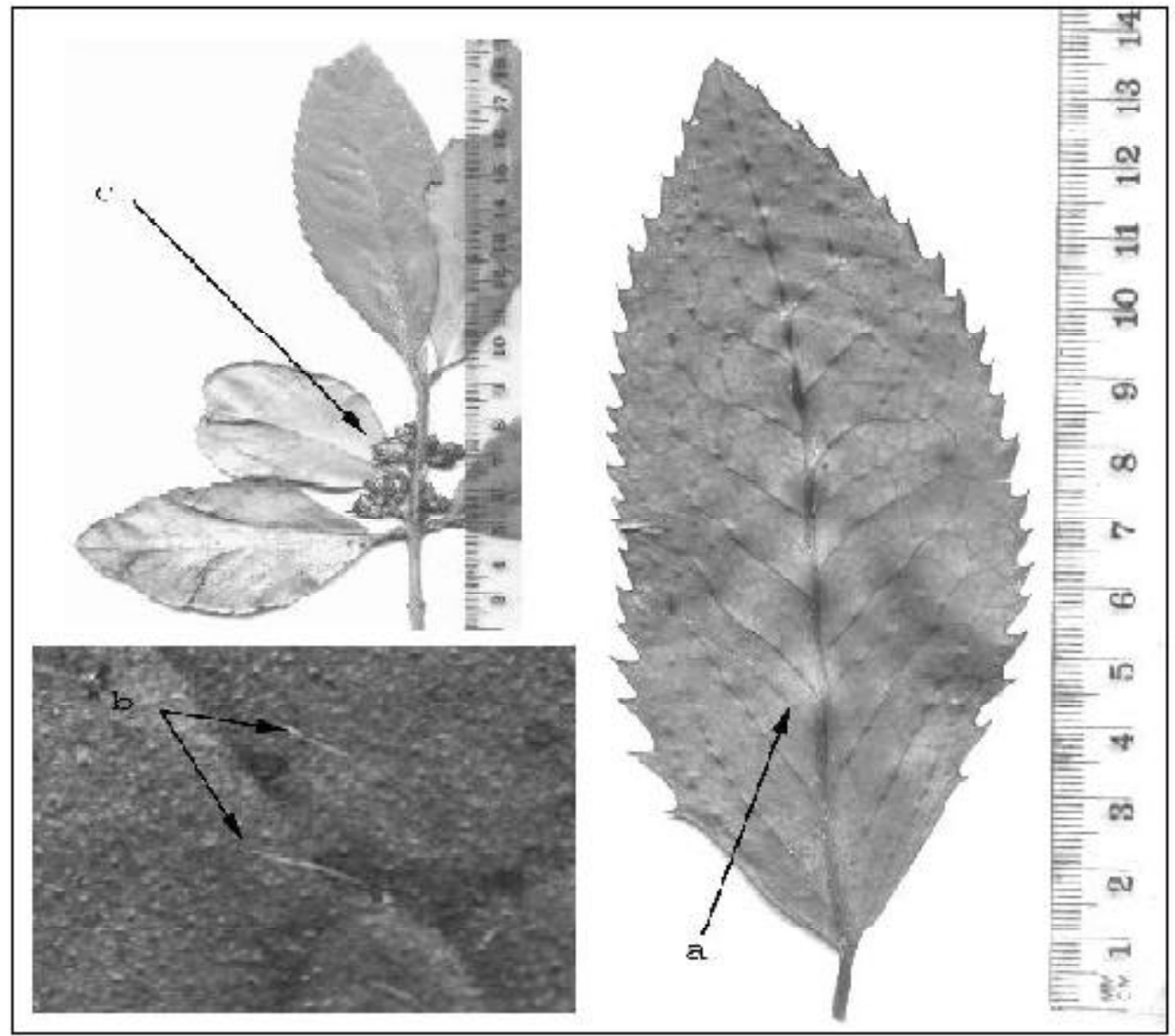

FIGURA 1. Hoja del ejemplar de Laureliopsis philippiana estudiado (a), pelos malpighiáceos de la misma (b) y ejemplar con frutos (c).

Material de estudio: Chile. Séptima Región. Provincia de Talca. San Pedro-Las Cañas. 410 msnm. $35^{\circ} 29^{\prime} \mathrm{S}$ y $72^{\circ} 22^{\prime}$ O. 19-VI-2003. César A. Sepúlveda s/n. Herbario de la Universidad de Talca $\mathrm{N}^{\circ} 2731$.

\section{AGRADECIMIENTOS}

Agradecemos las facilidades de acceso y ayuda en terreno de Alex Leiva y Leonardo Vergara, del Area de Biodiversidad de la empresa Forestal Copihue. A Patricio Peñailillo, José San Martín y Alexandra Stoll, de la Universidad Talca. A los comentarios de C. Lusk y un árbitro anónimo.

\section{BIBLIOGRAFIA}

Barrera, E. \& I. Meza. 1992. Características de la epidermis foliar de las especies chilenas I. Subclase Magnoliidae. Boletín Museo Nacional de Historia Natural Chile 43: 29-39.

Barrera, E. 1981. Análisis de la cutícula foliar de las especies chilenas del género Polypodium L. Boletín Museo Nacional de Historia Natural Chile 38: 21-28.

Arroyo, M.T.K.,L. Cavieres A. PeÑaloza, M. Riveros \& A.M. FAGGi. 1996. Relaciones fitogeográficas y patrones regionales de riqueza de especies en la flora del bosque lluvioso templado de Sudamérica. En: Ecología de los bosques nativos de Chile (Eds. J.J. Armesto, C. Villagrán \& M.T. K.Arroyo), pp. 71-100. Editorial Universitaria, Santiago, Chile. 
Rodríguez, R. \& M. Quezada. 2001. Monimiaceae. Flora de Chile. Vol 2 (1):10-15.

Sepúlveda, C.A\&A. Stoll. 2003. Presencia deNothofagus alpina (Poepp. et Endl.) Oerst. (Fagaceae) en el borde costero de la Región del Maule, Chile central. Gayana Botanica 60: 134-135.
Troncoso, A. \& J. SAN M ARTín. 1988. Ampliación de área para diversas especies de plantas vasculares en la Cordillera de la Costa de la Región del Maule. Boletín Museo Nacional de Historia Natural Chile 41: 45-56.

Fecha de recepción: 18.12.03

Fecha de aceptación: 08.01.04 